\section{Tips for youth}

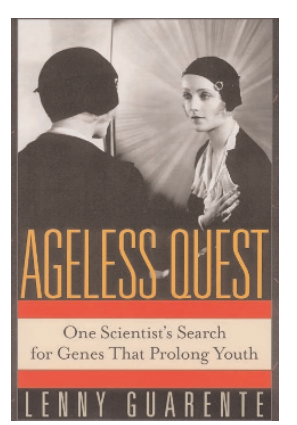

\section{Ageless Quest: One Scientist's Search for Genes that Prolong Youth}

by Lenny Guarente

Cold Spring Harbor

Laboratory Press, 2003

Hardback, \$19.95

Thomas B. L. Kirkwood

The idea that yeast has anything to tell us about the mysteries of human ageing might, at first sight, seem laughable. Human ageing is characterized by an array of changes affecting each of the various organ systems - brain, heart, skin, bone, blood vessels, gut, sensory organs, immune system, and so on - in subtly different ways. How can a single-celled organism represent a meaningful model for this complexity? Indeed, can the concept of 'ageing' be applied at all to a creature that reproduces simply by splitting in two?

What Lenny Guarente shows in Ageless Quest is that in at least some respects, yeast is a valuable model for ageing research and that the principles underlying the genetic regulation of its 'lifespan' are not as different from ours as might be expected. Recent advances in the biology of ageing across many fronts have clearly shown that the underlying cause of ageing seems to be the gradual accumulation of molecular damage. In this respect, the molecular biology of a yeast cell is not so different from that of a mammal. The same molecular targets for damage exist and the same biochemical processes generate the damage that affects them. Furthermore, we have found that the rate of molecular ageing is governed by the efficacy of the mechanisms for cellular maintenance and repair, and these are remarkably well conserved across vast phylogenetic ranges.

Finally, there is growing evidence that high-level regulators of cellular metabolism, such as insulin signalling pathways, influence the balance between maintenance and other functions, such as growth and reproduction. This form of regulation, which probably helps by providing a degree of adaptive plasticity to allow organisms to cope with fluctuating nutrient availability, seems to work across the species range.

These strands of our new understanding of the biology of ageing are woven together by the theory of the 'disposable soma', which recognizes

Thomas B. L. Kirkwood is in the Henry Wellcome Laboratory for Biogerontology Research, Institute for Ageing and Health, University of Newcastle, Newcastle upon Tyne, NE4 6BE, UK.

e-mail: Tom.Kirkwood@ncl.ac.uk. that organisms are under constant evolutionary pressure to optimize their allocation of resources between competing metabolic priorities. Given the mortality pressure exerted by the environment, individual organisms tend to die young. It therefore makes no sense to invest greater effort in maintenance of the individual body, or soma, than is needed to stay in good shape for as long as the individual can reasonably expect to evade these extrinsic dangers. From the evolutionary perspective, it is better to use any surplus for growth and reproduction. This explains a lot about why and how we age, but has it any relevance for single-cell yeast?

Central to Guarente's story is the gene SIR2, where SIR stands for silent information regulator. One of a troika of unrelated SIR proteins, the SIR2 gene product is involved in suppressing gene transcription. It also has a central role as a regulator of yeast lifespan, as measured by the number of divisions that a yeast mother cell could attain. At first, it seemed that SIR2 was involved merely in a specific form of genome instability: the formation of extrachromosomal rDNA circles. These could contribute to ageing, but it seemed unlikely that they represented any kind of general mechanism. However, it turned out that SIR2 has a bigger role as an NAD-dependent histone deacetylase, and it is in this role that it seems to function as a much more fundamental regulator of survival. Furthermore, through its close association with NAD, SIR2 is well-placed to respond to variations in environmental nutrient supply and to optimise the trade-off between survival and reproduction, just as the disposable soma concept suggests. But in a unicellular organism, surely Weismann's germline-soma distinction vanishes. Or does it? The essence of the Weismannian distinction is a division of labour, placing the responsibility for long-term continuity of genetic inheritance on some cells, but not on others.

The asymmetry of division in budding yeast between the large mother cell and the small daughter cell is coupled with the fact that mother cells age and die after a finite number of divisions, whereas the lineage of new daughters cells is 'immortal'. Guarente's work not only shows neatly how yeast is an exception that proves the rule, but also demonstrates how its very simplicity can help throw light on fundamental ageing processes in more complex organisms.

Guarente's book should be read widely as an accessible account of the science of ageing, but what makes this book exceptional is its portrayal of another 'ageless quest' - the pursuit of science itself. Nobel laureate Peter Medawar once wrote a book called Advice to A Young Scientist. In spite of the authority given to Medawar's book by his glittering achievements and matchless literary style, Advice to A Young Scientist did not give me the same sense of a life in science as does Ageless Quest. The glimpses of Guarente's life outside the lab give a real feel of the struggle for 'work-life balance', especially in the fraught pre-tenure stages of a career. The need to take risks comes across well. "You're gonna what?!" is the reaction of his department chairman to the news that Guarente is going to turn his lab towards the thorny problem of ageing. The highs and lows of doing cutting-edge research in a young field of research come alive, as does the importance of teamwork. My advice to a young scientist would be simple: read Ageless Quest. 